\title{
Uma Análise Da Educação Em Saúde Na Redução Dos Indicadores De Infecção Primária Relacionada A Cateter Venoso Central
}

\author{
An Analysis Of Health Education In The Reduction Of Indicators Of Primary Infection Related To \\ Central Venous Cateter
}

\section{Análisis De La Educación Para La Salud En La Reducción De Indicadores De Infección Primaria Relacionados Con El Cateter Venoso Central}

Joseane Stahl Silveira ${ }^{1}$ Rita de Cassia Nugem ${ }^{2}$

Resumo: As infecções hospitalares constituem grave problema de saúde pública. O objetivo deste estudo foi verificar de que forma a Educação Permanente em Saúde pode contribuir para a diminuição dos indicadores de infecção primária relacionada ao cateter venoso central em um hospital público e universitário da cidade de Porto Alegre, Rio Grande do Sul. Foram empregados dados referentes aos indicadores de infecção primária relacionada ao cateter venoso central e às ações de educação com o mesmo tema, de informações dos Relatórios de Indicadores

\footnotetext{
${ }^{1}$ Profissional da área de gestão de pessoas e educação em saúde. Graduada em Pedagogia Empresarial (Ulbra). Especialista em: Psicologia do Trabalho e das Organizações (Feevale); Gestão do Trabalho e Educação em Saúde (Fiocruz) e Gestão em Saúde (Ufrgs). Analista de Qualificação e Aperfeiçoamento no Hospital de Clinicas de Porto Alegre. E-mail: jstahl@hcpa.edu.br. https://orcid.org/0000-0002-9932-2514

${ }^{2}$ Professora e pesquisadora das áreas de administração em saúde e saúde pública. : Doutoranda em Administração (UFRGS), Doutoranda em Saúde Pública (UCB-Lyon1); Mestre em Saúde Coletiva (UFRGS); Especialista em Educação e Tecnologias EAD (UNICID); Graduada em Administração de Sistemas e Serviços de Saúde (UERGS). Doutoranda e Pesquisadora - Universidade Federal do Rio Grande do Sul e Université Claude Bernard Lyon 1 - France. E-mail: rita-de-cassia.nugem@univ-lyon1.fr. https://orcid.org/0000-0002-8713-0187
}

de Infecção Hospitalar, dos Relatórios de Gestão e Administração e das Querys (banco de dados) de capacitação, relativas aos anos de 2014 a 2017. Os resultados mostram que o indicador de infecção apresentou redução de 4,21 para 3,0 infecções por 1000 procedimentos-dia nos referidos anos. As ações educativas relacionadas ao tema do cateter venoso central aumentaram no período, elevando o número de participantes e participações nas atividades (quatro vezes mais) em 2017. Houve também um público alvo mais abrangente, onde a enfermagem representou $38 \%$ dos profissionais capacitados, seguida pelos médicos $(31 \%)$ e técnicos em enfermagem (25\%). As capacitações realizadas, juntamente com as outras ações, auxiliaram na redução dos indicadores de infecção por cateter venoso central.

Palavras-chave: Educação em Saúde. Infecção Hospitalar. Gestão em Saúde. Cateteres de demora. Capacitação em Serviço. 
Abstract: Hospital infections are a serious public health problem. The objective of this study was to verify how Permanent Health Education can contribute to the reduction of indicators of primary infection related to the central venous catheter in a public and university hospital in the city of Porto Alegre, Rio Grande do Sul. the indicators of primary infection related to the central venous catheter and the educational actions with the same theme, information from the Hospital Infection Indicator Reports, the Management and Administration Reports and the Querys (database) of training, related to the years from 2014 to 2017 . The results show that the indicator of infection presented reduction from 4.21 to 3.0 infections per 1000 procedures per day in those years. The educational actions related to the central venous catheter issue increased in the period, increasing the number of participants and participation in activities (four times more) in 2017. There was also a more comprehensive target audience, where nursing represented $38 \%$ of trained professionals, followed by physicians $(31 \%)$ and nursing technicians $(25 \%)$. The training performed, along with the other actions, helped reduce the indicators of central venous catheter infection.

Key words: Health Education. Hospital Infection. Health Management. Catheters of delay. Training in Service.

Resumen: Las infecciones hospitalarias son un grave problema de salud pública. El objetivo de este estudio fue verificar cómo la Educación para la Salud Permanente puede contribuir a la reducción de los indicadores de infección primaria relacionada con el catéter venoso central en un hospital público y universitario en la ciudad de Porto Alegre, Rio Grande do Sul. los indicadores de infección primaria relacionados con el catéter venoso central y las acciones educativas con el mismo tema, información de los Informes de indicadores de infecciones hospitalarias, los Informes de administración y administración y las consultas (base de datos) de capacitación, relacionadas con los años de 2014 a 2017. Los resultados muestran que el indicador de infección presentó una reducción de 4.21 a 3.0 infecciones por 1000 procedimientos por día en esos años. Las acciones educativas relacionadas con el tema del catéter venoso central aumentaron en el período, lo que aumentó el número de participantes y la participación en actividades (cuatro veces más) en 2017. También hubo un público objetivo más amplio, donde la enfermería representó el $38 \%$ de los profesionales capacitados. por médicos (31\%) y técnicos de enfermería (25\%). El entrenamiento realizado, junto con las otras acciones, ayudó a reducir los indicadores de infección del catéter venoso central.

Palabras clave: Educación para la salud. Infección hospitalaria. Gestión de la salud. Catéteres de retraso. Entrenamiento en Servicio. 


\section{Introdução}

Vários são os problemas atuais do sistema de saúde brasileiro, em suas diversas instâncias. A área hospitalar é constantemente desafiada pelas infecções hospitalares, que constituem grave problema de saúde pública mundial, aumentando a mortalidade entre os pacientes e elevando os custos hospitalares ${ }^{(1)}$.

A redução das taxas de infecção hospitalar é sempre uma meta a ser atingida. É um dos fatores prioritários para promover a segurança do paciente e a qualidade assistencial. A análise destes indicadores é necessária a fim de se buscar medidas de controle em tempo hábil, a serem tomadas tanto pelas instituições, quanto pelos órgãos orientadores $^{(2)}$.

Dentre as causas mais frequentes de infeção hospitalar, estão às infecções primárias de corrente sanguínea (IPCS). Estima-se que cerca de $60 \%$ sejam associadas a algum dispositivo intravascular. A IPCS associa-se a um aumento na taxa de mortalidade, a um maior tempo de internação e a incrementos de custos relacionados à saúde ${ }^{(3)}$.

A Política de Educação Permanente em Saúde (PEPS), foi oficializada na Constituição Federal de 1988, mas instituída de fato em 2004, com a portaria 198/2004, como estratégia do Sistema Único de Saúde para a formação e o desenvolvimento de seus trabalhadores. Preconiza a capacitação dos profissionais a partir da realidade em que estão inseridos e prioriza a transformação desta realidade. Porém, para efetivarmos a PEPS, é preciso analisar seus resultados junto ao processo de assistência ao paciente. Santos $^{(1)}$, em sua pesquisa realizada em hospitais, aborda que a incorporação de ações de prevenção e controle de infecções hospitalares ocorreu de forma heterogênea entre as instituições estudadas, mas que ações mais complexas foram menos presentes nas respostas, como por exemplo, desenvolvimento de programas de controle de infecções (49\%), treinamentos específicos em controle de infecções (44\%) e adoção de medidas de contenção de surtos (33\%).

O objetivo geral desta pesquisa foi comparar os indicadores de infecções relacionadas ao cateter venoso central, em um Hospital Universitário de Porto Alegre, com as atividades educativas realizadas de mesmo tema, entre os anos de 2014, ano em que o indicador de cateter foi alterado conforme regulamentação da Anvisa, a 2017, ano este em que foi alcançado o menor índice até então, para verificar de que forma a educação em saúde pode contribuir para a melhoria dos processos assistenciais de saúde, nesse caso, para a diminuição de infecção primária relacionada ao cateter venoso central.

\section{Objetivos}

O objetivo desta pesquisa é analisar a relação entre o indicador de infecção do cateter venoso central e as atividades educativas realizadas em um hospital 
universitário, da cidade de Porto Alegre, Rio Grande do Sul (RS), entre os anos de 2014 a 2017.

Para isso, é necessário descrever o indicador de Infecção Primária Relacionada ao Cateter Venoso Central, presente nos Relatórios de Gestão e Administração Anual da instituição, identificar a quantidade, datas de realização e o número de participantes nas capacitações realizadas quanto ao tema de Cateteres Venosos Centrais e, por fim, comparar os indicadores de infecção primária relacionada ao cateter venoso central com as capacitações realizadas.

\section{Revisão De Literatura}

3.1 Política de Educação Permanente em Saúde

A educação por muito tempo se caracterizou pela fragmentação disciplinar e pela separação das áreas de conhecimento, com base em metodologias tradicionais de transmissão de conhecimento, em um currículo separado por disciplinas. E o ensino em saúde não poderia ser diferente, sendo, na maioria dos casos, as capacitações baseadas na transmissão de conhecimentos dentro da lógica do "modelo escolar".

A trajetória de estruturação do SUS é acompanhada de perto também pela necessidade de organização da educação em saúde, pois a saúde e a educação caminham juntas, frente às constantes necessidades de atualização e aprendizado na assistência. Mas é na Constituição de 1988 que a Política de
Educação Permanente em Saúde é oficializada, sendo consolidada em 2004 com a portaria 198/2004, do Ministério da Saúde.

Ela tem por objetivo problematizar a gestão do trabalho dos profissionais de saúde, contribuindo na melhoria dos processos de trabalho e visa orientar a formação e o desenvolvimento dos trabalhadores, nestas novas perspectivas. Ela deve ser realizada pelos polos de educação permanente, com a função de identificar as necessidades de formação e desenvolvimento dos trabalhadores, e construir alternativas para a atenção e a gestão e, fortalecimento do controle social $^{(4)}$.

É responsabilidade dos Colegiados de Gestão Regional, com a participação das Comissões Permanentes de Integração Ensino-Serviço (CIES), elaborar um Plano de Ação Regional de Educação Permanente em Saúde, coerente com os Planos de Saúde estadual e municipais, referente à educação na saúde. Devem considerar as especificidades regionais, as necessidades de formação e o desenvolvimento para o trabalho e as ações formais de educação na saúde ${ }^{(5)}$.

Atualmente, a Política de Educação Permanente em Saúde esta além dos polos de educação, sendo norteadora de todas as esferas da área saúde, orientando as atividades que visam uma melhor qualificação dos trabalhadores ${ }^{(6)}$

Mais do que apenas a capacitação, a PEPS representa uma valorização de seus colaboradores. A escolha da Educação 
Permanente em Saúde como ato político de defesa do trabalho no Sistema Único de Saúde (SUS) decorreu do desafio para que o setor da saúde correspondesse às necessidades da população, conquistasse a adesão dos trabalhadores, constituísse processos vivos de gestão participativa e transformadora e seduzisse docentes, estudantes e pesquisadores a mais vigorosa implementação do SUS ${ }^{(6)}$.

Educação Permanente, em suas definições, significa $\mathrm{o}$ atendimento às necessidades de formação e qualificação sistemática e continuada dos trabalhadores do SUS. É um processo de constante aprendizado do trabalhador, de qualquer conhecimento, por meio de educação formal ou não formal. Tem o objetivo de melhorar as atividades do trabalhador, levando em consideração as suas necessidades, da equipe de trabalho e da instituição em que trabalha, dos usuários e da demanda social $^{(5)}$.

É a aprendizagem no cotidiano do trabalho, onde o aprender e o ensinar se incorporam a rotina da organização. Possui como base a aprendizagem significativa, realizada a partir dos problemas da realidade e considera no processo de aprendizagem, os conhecimentos e as experiências que as pessoas já possuem anteriormente, com o objetivo de transformar a organização do trabalho e melhorar os resultados da assistência $^{(5)}$.

\subsection{Infecção Hospitalar e seus} indicadores
Infecção Hospitalar (IH) é a infecção adquirida durante a hospitalização. São consideradas as principais causas de morbidade e de mortalidade, além de aumentarem o tempo de internação do paciente, elevando o custo do tratamento. Para promover a segurança do paciente e garantir a qualidade assistencial, é muito importante controlar as taxas de infecção hospitalar. É necessário estabelecer e analisar estes indicadores, para acompanhamento e busca de medidas de controle em tempo hábil (2).

Quando as instituições de saúde conhecem seus problemas de infecção, através dos indicadores e outros índices de medição, se torna maior a adesão das equipes aos programas de prevenção e controle. Pesquisas apontam que pode ocorrer uma redução de até $70 \%$ para algumas infecções, como por exemplo, para as infecções da corrente sanguínea ${ }^{(7)}$.

Um estudo da Organização Mundial de Saúde (OMS) demonstrou que a maior prevalência de infecções ocorre em unidades de terapia intensiva, em enfermarias cirúrgicas e alas de ortopedia, sendo que as infecções mais frequentes são as de sítio cirúrgico, trato urinário e trato respiratório inferior $^{(7)}$.

3.2.1 Infecção relacionada ao cateter venoso central

O uso de cateter começou em hospitais nos anos 40, tornando-se essencial 
para as atividades assistenciais. Embora esse dispositivo facilite 0 tratamento, principalmente de pacientes internados, pois permite um acesso rápido à corrente sanguínea, a sua utilização está relacionada a elevadas taxas de mortalidade e de custos hospitalares ${ }^{(8)}$.

$\mathrm{O}$ cateter venoso central (CVC) "é um sistema intravascular utilizado para fluidoterapia, administração de fármacos, infusão de derivados sanguíneos, nutrição parenteral, monitorização hemodinâmica, terapia renal substitutiva, entre outros. É um dispositivo que pode permanecer no paciente por vários dias, minimizando o trauma associado às repetidas inserções de um cateter venoso periférico" ${ }^{(9)}$.

Porém falhas técnicas na sua utilização acontecem com frequência, aumentando as taxas de infecção de corrente sanguínea (ICS). Por isso, a definiçãa de estratégias e procedimentos para correção dessas falhas tem sido um desafio às equipes assistenciais ${ }^{(9)}$.

Conforme novas orientações da Anvisa, "a partir de janeiro de 2014, todos os serviços de saúde com qualquer número de leitos de Unidade de Terapia Intensiva (UTI) passaram a ter, obrigatoriamente, que notificar mensalmente seus dados sobre IPCS relacionadas ao uso de CVC em UTI e marcadores de resistência microbiana relacionados a estas infecções, além de notificar Infecções de Sítio Cirúrgico (ISC) relacionadas ao parto cirúrgico: cesariana"(10).
Um estudo de revisão reportou que a incidência de infecção relacionada ao CVC pode variar de 3,2 a 40,4 casos por 1.000 dias de cateter. A taxa de mortalidade dos pacientes oscilou entre 6,7 e 75\%. Estima-se que cerca de $60 \%$ das infecções relacionadas à saúde sejam associadas a algum dispositivo intravascular. Desse modo, esforços para distinguir as taxas de IPCS contribuirão para a segurança dos pacientes e qualidade da assistência $^{(9)}$.

No Brasil, conforme dados da ANVISA, "ainda há poucos estudos que avaliem o impacto econômico destas infecções, no entanto, dados preliminares sugerem que ele varie entre 7.906 dólares americanos a 89.866 dólares por episódio"(10).

A não realização da educação permanente em saúde dos profissionais é citada, junto a outros fatores assistenciais, como a não realização de técnicas corretas e o descumprimento de normas, como principais no aumento do risco das infecções em instituições de saúde ${ }^{(9)}$.

Estudos recentes relatam a redução das taxas de infecção após a adoção de estratégias educacionais sobre a inserção e a manutenção com os cateteres, onde a mudança no processo de trabalho da equipe é o objetivo ${ }^{(10)}$.

\section{Metodologia Da Pesquisa}

Essa pesquisa se caracteriza como quantitativa e descritiva através da coleta e análise de dados secundários de cunho documental. A abordagem quantitativa 
caracteriza-se pelo emprego da quantificação tanto na coleta de informações, quanto no tratamento dos dados através de recursos matemáticos ou estatísticas ${ }^{(11)}$.

Neste trabalho, analisaram-se os dados sobre as infecções relacionadas ao cateter venoso central, as capacitações realizadas e suas possíveis contribuições na redução desta infecção, entre os anos de 2014 a 2017. O ano de 2014 foi considerado como base, pois houve uma mudança no indicador, para adequação aos critérios da ANVISA. E o ano de 2017, foi o período que apresentou os menores índices de infecção por cateter venoso até então mensurados.

Este estudo foi desenvolvido em um hospital universitário, da cidade de Porto Alegre, Rio Grande do Sul (RS). É um hospital terciário, inserido nas redes nacional, estadual e municipal da saúde, com uma capacidade instalada de 839 leitos.

A coleta de dados do indicador de cateter foi realizada através dos Relatórios de Gestão e Administração e dos Relatórios dos Indicadores de Infecção Hospitalar. Para análise das capacitações de cateter venoso central, foram emitidas Querys do sistema de recursos humanos. A Queryé um processo de extração de informações de um banco de dados e sua apresentação em forma adequada ao uso. A partir da geração das Querys, foram aplicados filtros para análise e levantamento das capacitações, tais como: Nome do Evento (cateter, infecção, IPCS e Cateter Venoso Central de Inserção Periférica de Longa Permanência (PICC)); tipo de participação (participante); ambiente de realização (internas, externas e em EAD); período de realização (data fim do evento, acontecendo em 2014 e 2017). Após o filtro inicial fez-se a análise do que era referido ao cateter venoso central e outros cateteres, desde que abrangessem o central também.

\section{Resultados E Discussão}

5.1 Indicadores de Infecção Primária Relacionada a Cateter Venoso Central

O Relatório de Gestão de Exercício do hospital tem o objetivo de prestar contas aos órgãos de controle, no que se refere às atividades realizadas pela instituição no exercício do ano, onde é descrito o planejamento organizacional e os resultados alcançados ao longo de cada ano ${ }^{(12)}$.

A infecção primária de corrente sanguínea relacionada ao cateter venoso central é um dos indicadores de processos internos do planejamento estratégico ${ }^{(13)}$

Abaixo, um quadro com a evolução do indicador nestes anos e as ações realizadas para a redução destas infecções.

\section{Tabela 1: Indicador de Infecção Primária de Corrente Sanguínea Relacionada ao Cateter Venoso Central (IPCS) de 2014 a 2017}




\begin{tabular}{|c|c|c|c|}
\hline \multirow[b]{2}{*}{ Ano } & Meta & Resultado & \multirow[b]{3}{*}{$\begin{array}{l}\text { Ações } \\
\text { Manutenção da recomendação de passagem de cateteres venosos } \\
\text { centrais no centro cirúrgico ambulatorial; Estabelecimento de metas } \\
\text { individuais por unidades; Intervenção específica na unidade de } \\
\text { ambiente protegido; disponibilização do uso de curativos } \\
\text { impregnados com clorexidina }{ }^{(13)} \text {. }\end{array}$} \\
\hline & \multicolumn{2}{|c|}{$\begin{array}{c}\text { (infecções / 1000 } \\
\text { procedimentos-dia) }\end{array}$} & \\
\hline 2014 & $\leq 2,0$ & 4,21 & \\
\hline 2015 & $\leq 3,5$ & 3,20 & $\begin{array}{l}\text { Instituição de ações corretivas de capacitação e feedback de } \\
\text { resultados; O monitoramento da realização de um pacote de medidas } \\
\text { preventivas para prevenção da infecção nas unidades com maiores } \\
\text { taxas de infecção; A recomendação de uso de paramentação } \\
\text { completa para passagem de cateter e o uso do Centro Cirúrgico } \\
\text { Ambulatorial como local de eleição para passagens de cateteres } \\
\text { eletivos de pacientes adultos }{ }^{(14)} \text {. }\end{array}$ \\
\hline 2016 & $\leq 3,0$ & 3,30 & $\begin{array}{l}\text { Mudança da rotina de desinfecção das conexões dos cateteres nas } \\
\text { unidades de Ambiente Protegido e Oncopediatria e monitoramento e } \\
\text { retorno às unidades desse processo; Projeto piloto e introdução de } \\
\text { sachês com álcool para desinfecção de cateteres nas unidades de } \\
\text { Pediatria, Internação clinica e Internação Cirúrgica e ampliação do } \\
\text { uso desses sachês para as demais unidades }{ }^{(12)}{ }^{\text {. }}\end{array}$ \\
\hline 2017 & $\leq 3,0$ & 3,00 & $\begin{array}{l}\text { Criação do protocolo de uso de cateteres venosos; Disponibilização } \\
\text { do curso em EAD para o treinamento do manejo destes cateteres; } \\
\text { Educação presencial sobre o cuidado na manutenção dos cateteres } \\
\text { em parceria com o Serviço de Educação em Enfermagem; Ações e a } \\
\text { criação do dia do cateter - para sensibilização dos profissionais; Foco } \\
\text { das ações nessas unidades com a maior taxa de cateteres, no que se } \\
\text { referem a treinamentos, indicadores e acompanhamento das ações }{ }^{(15)} \text {. }\end{array}$ \\
\hline
\end{tabular}

\section{Fonte: autoria própria (2019).}

5.2 Ações Educativas Sobre os Cateteres Venosos Centrais

O Plano de Educação e

Desenvolvimento de Pessoas da instituição em estudo visa definir as ações de desenvolvimento dos colaboradores, contribuindo para a excelência na prestação de serviços de forma a garantir a melhor qualidade assistencial e o alinhamento com as políticas públicas de saúde. Tem como objetivo instituir, implementar e regulamentar as ações para educação e desenvolvimento de pessoas, respeitando a legislação vigente.

O Plano delimita que a Educação e o Desenvolvimento de Pessoas estão alinhados à PEPS (Portaria nº 1996/07), que tem como objetivos a transformação das práticas profissionais e da própria organização do trabalho, a partir da problematização deste e da aprendizagem significativa. Diz que as necessidades de capacitação e 
desenvolvimento são identificadas de acordo com as definições estratégicas da Instituição (Planejamento Estratégico), os projetos e programas institucionais, os indicadores de qualidade assistencial, as alterações de processos ou adoção de novas tecnologias, bem como através de demandas pontuais das áreas.

O indicador de infecção de cateter venoso central faz parte do planejamento estratégico do hospital, sendo uma meta acompanhada mensalmente. Este indicador serve para auxiliar na análise da qualidade assistencial e de melhorias nos processos de trabalho, tornando-se assim prioridade nas ações de capacitação ${ }^{(12)}$.

Abaixo, na Tabela 2, as informações sobre as ações educativas realizadas, apresentando o número de participações e o público-alvo.

Tabela 2: Ações Educativas de Cateter Venosos Central de 2014 a 2017

\begin{tabular}{|c|c|c|c|c|}
\hline Ano & Ações & Participantes & Participações & Público - Alvo (participantes) \\
\hline 2014 & 20 & 296 & 408 & $\begin{array}{l}\text { - Enfermeiros: } 144(48,65 \%) \\
\text { - Técnicos em enfermagem: } 93 \\
(31,42 \%)\end{array}$ \\
\hline 2015 & 21 & 303 & 347 & $\begin{array}{l}\text { - Técnicos em enfermagem: } 131 \\
(43,23 \%) \\
\text { - Enfermeiros: } 106(34,98 \%)\end{array}$ \\
\hline 2016 & 25 & 401 & 509 & - Enfermeiros: $359(89,52 \%)$ \\
\hline 2017 & 28 & 1418 & 2037 & $\begin{array}{l}\text { - Enfermeiros: } 537(37,87 \%) \\
\text { - Médicos: } 438 \text { (30,89\%) } \\
\text { - Técnicos em enfermagem: } 359 \\
(25,32 \%)\end{array}$ \\
\hline
\end{tabular}

Fonte: autoria própria (2019).

\subsection{Indicador de Infecção e as Ações Educativas Realizadas}

Comparando o indicador de cateter entre 2014 e 2017, é possível verificar uma redução de mais de $28 \%$, passando de 4,21 para 3,00 infecções por 1000 pacientes-dia. Veja no gráfico abaixo

\section{Gráfico 1: Evolução do Indicador de Infecção de Cateter Venoso Central por 1000 procedimentos-dia dos anos de 2014 a 2017}




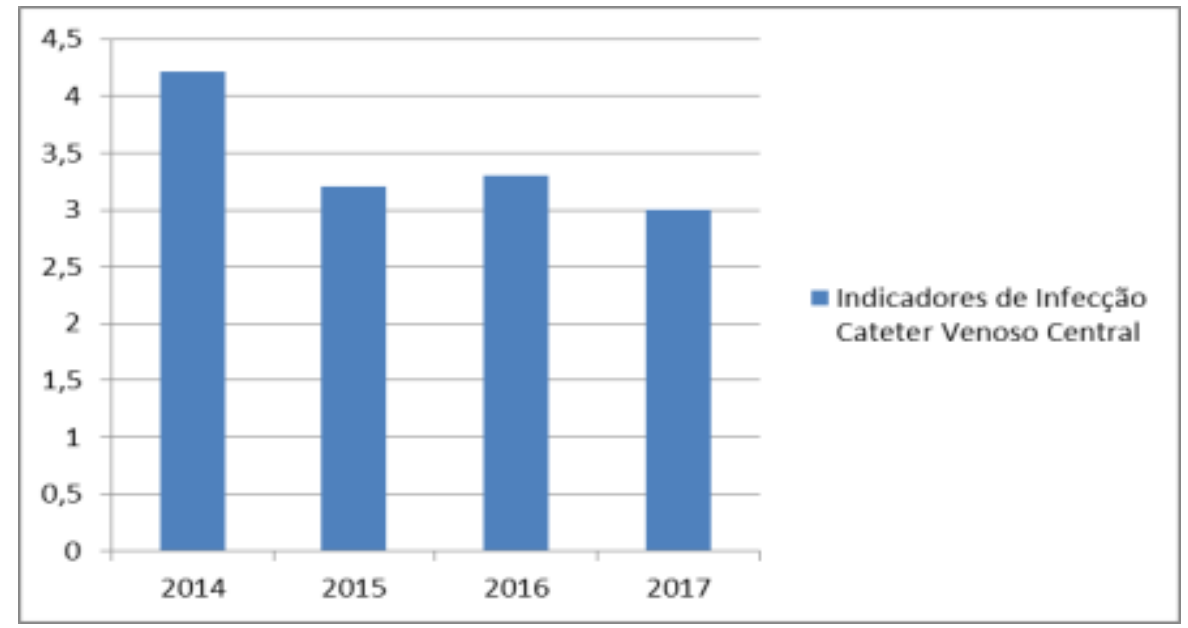

Fonte: autoria própria (2019).

As capacitações realizadas nos mesmos anos, relativas ao tema de Cateter, apresentam um aumento em 2017, comparado aos anos anteriores, quadruplicando as participações e os participantes.

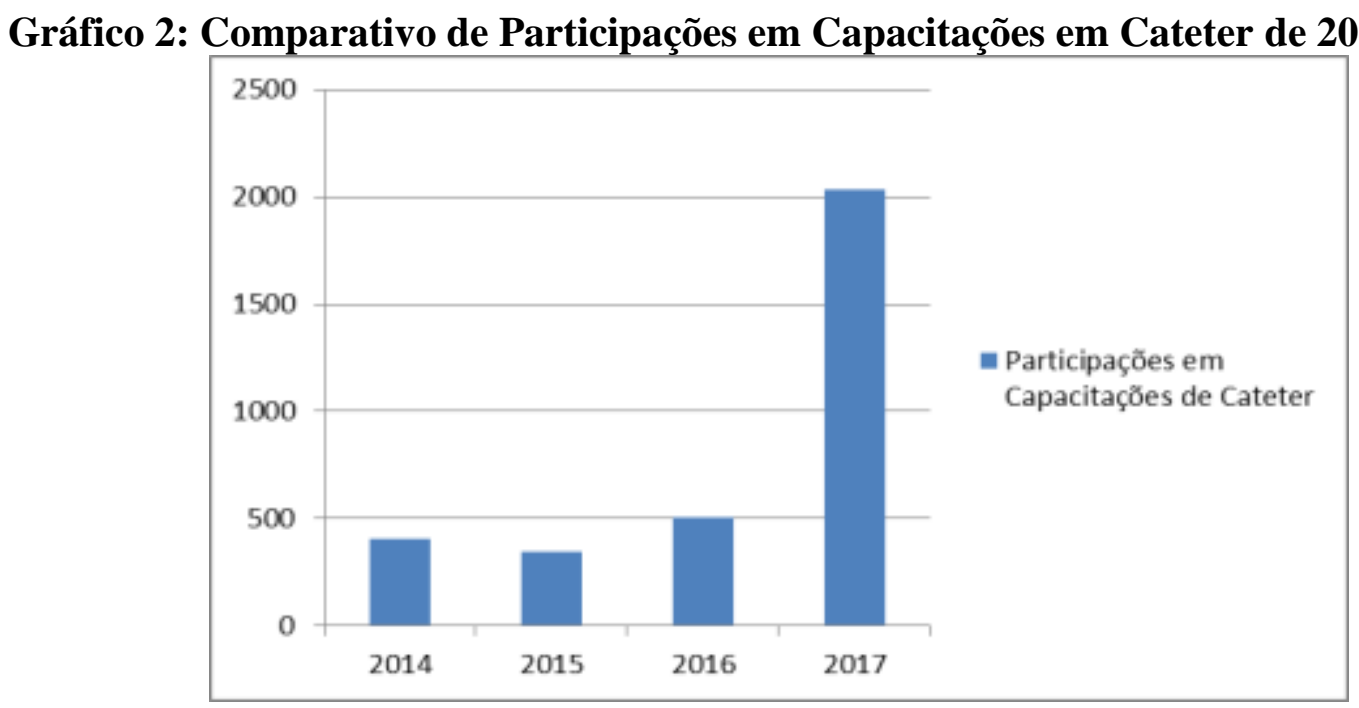

Fonte: autoria própria (2019).

Conforme o Gráfico 3, no resultado do indicador de cateter de 2017, observa-se que as taxas se mantiveram acima da meta em janeiro, fevereiro e março, ficando abaixo nos demais meses. Comparado com as Abaixo o comparativo dos resultados do indicador de cateter e as capacitações realizadas em mesmo período. Rev. Gestão \& Saúde (Brasília) Vol. 10, n. 03, Set. 2019. capacitações no mesmo período, verifica-se que a partir do mês de março cresce o número de participações nos eventos, mantendo-se alto até o final do ano, quando novamente apresenta um aumento nas participações. 
Gráfico 3: Comparativo indicador de cateter e participações de capacitação de 2016 e 2017

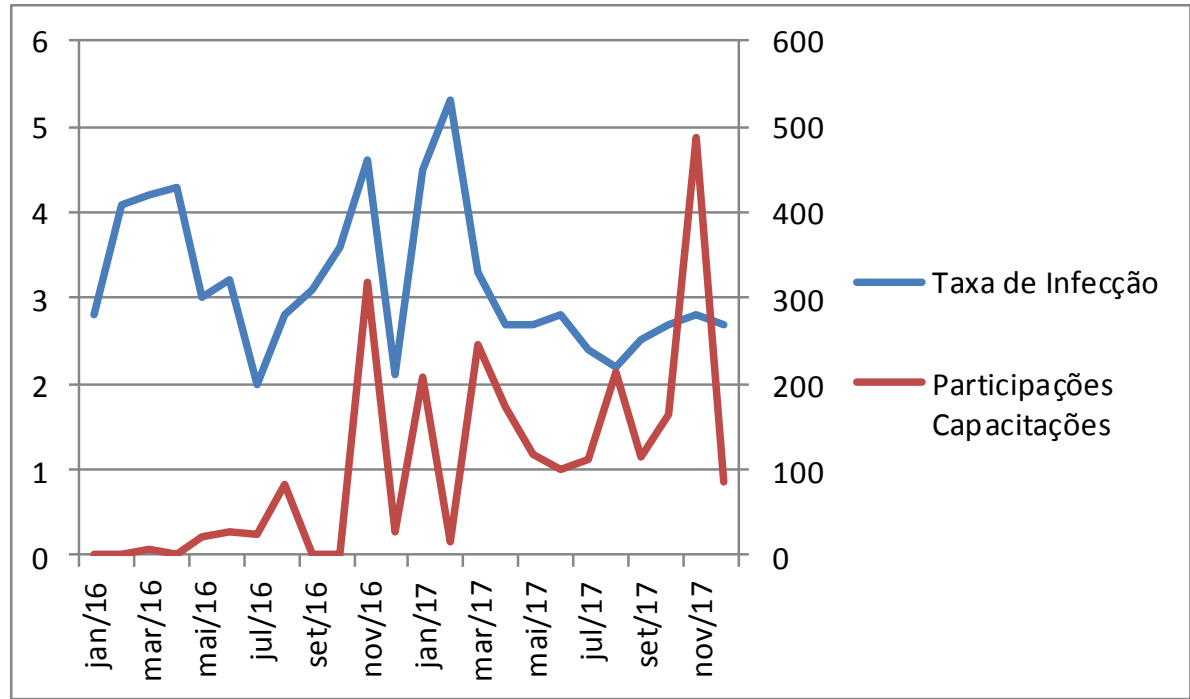

Fonte: autoria própria (2019).

Ao comparar estes resultados, se percebe que a redução do indicador a partir de abril de 2017 acontece concomitantemente com o aumento nas participações de capacitações em março do mesmo ano, que mantem a média maior durante o restante do ano.

Abaixo o comparativo dos indicadores de cateter e das capacitações realizadas de 2014 a 2017.

\section{Gráfico 4: Comparativo do indicador de cateter e das participações em capacitações de 2014 a} 2017

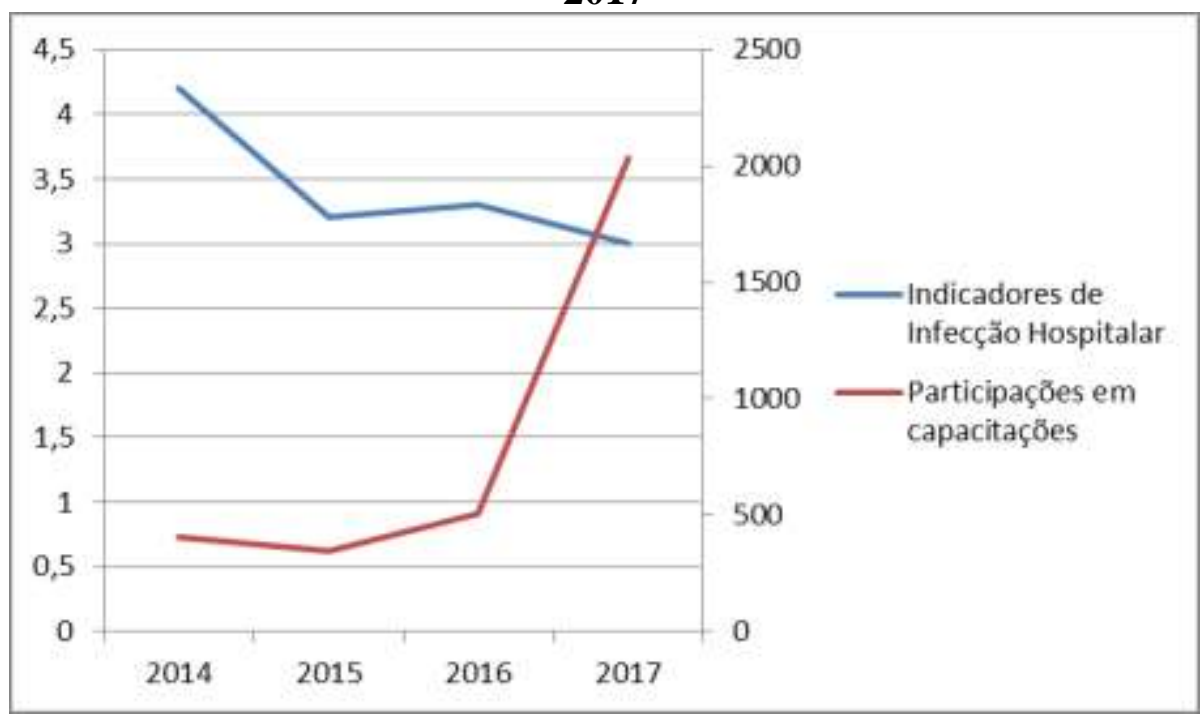

Fonte: autoria própria (2019).

A melhoria no indicador, conforme o relatório de gestão do ano de 2017, foi atribuída a várias ações que foram implementadas, entre elas estão: o início do curso em Educação a Distância (EAD); o lançamento do Protocolo Assistencial de 
Passagem de Cateter Venoso Central e Periférico; o treinamento de residentes médicos; as ações da Comissão de Cateteres na Hemodiálise; a abertura da sala de passagem de cateter venoso central para pacientes adultos; a divulgação de indicadores e do protocolo assistencial; as capacitações de enfermeiros sobre os cuidados com os cateteres venosos $^{(15)}$.

No mês de março de 2017 é importante destacar o lançamento do curso EAD de "Cateteres Venosos Centrais: indicação, inserção, manutenção e retirada", que por sua modalidade, facilita a participação e a realização da capacitação, além de ser uma atividade multidisciplinar, que abrangia diferentes categorias em seu público alvo.

Ou seja, tanto através do comparativo dos indicadores, que mostram a evolução do indicador de cateter venoso central concomitante com o aumento das capacitações, quanto no descrito pela instituição em seu relatório de gestão, as ações de educação foram importantes para a melhoria dos resultados na assistência.

Conforme Oliveira ${ }^{(16)}$, a educação permanente e treinamento da equipe de saúde são estratégias fundamentadas. Intervenções educativas na inserção do cateter por meio de simulação, programas de formação de enfermeiros em cuidados com o cateter ("equipes de terapia intravenosa") também foram associados com redução das ICSRC, em um estudo americano.

Outra análise importante a ser realizada é em relação ao público-alvo atingido. A maioria dos capacitados, de 2014 a 2017, é da área da enfermagem, isso porque são eles que atuam ininterruptamente na assistência e representam, na maioria das instituições hospitalares, o maior percentual dos trabalhadores. Além disso, são os profissionais que, direta ou indiretamente, estão mais envolvidos na implantação e manuseio de acesso vascular e consequentemente, com maior possibilidade de atuação na profilaxia e controle das infecções relacionadas ${ }^{(8)}$.

Porém, em 2017, ao haver uma maior participação também de outros profissionais, como os médicos, evidencia-se uma melhora nos resultados dos processos de cuidado. Conforme o que nos traz Mendonça (8), a designação de uma equipe multi e interdisciplinar específica e capacitada para inserção e manutenção de cateteres, seguindo protocolos padronizados pela $\mathrm{CCIH}$, pode levar à diminuição das taxas de ICS associada a uma melhor qualidade da assistência.

O resultado é de que a redução dos indicadores de infecção do cateter venoso central em 2017, se deu por diversos fatores, estando entres eles o aumento das ações educativas realizadas, com a abrangência de uma equipe multidisciplinar, além de outras 
ações assistenciais e de gestão realizadas, como já mencionado anteriormente.

\section{Conclusão}

A partir da análise dos relatórios de gestão foi possível constatar a redução dos indicadores de infecção, entre os anos de 2014 e 2017, de 4,21 infecções para 3,0 infecções por 1000 pacientes-dia, o menor da instituição.

Conforme o relatório de Gestão Anual da instituição, várias ações foram implantadas em 2017 para redução do indicador de infecção, entre elas são citadas diversas ações educativas, diferente dos anos anteriores, onde $\mathrm{o}$ foco eram ações assistenciais.

A partir disso então, ao se verificar as capacitações realizadas no período, foi possível constatar que em 2017 houve um aumento nas capacitações realizadas e principalmente no número de participantes e participações destas ações educativas, em média quatro vezes mais comparadas ao realizado nos anos anteriores, além de um público alvo mais abrangente.

Os resultados mostram uma redução de 2014 para 2017, em 28,74\% no indicador de cateter venoso central, ano em que quadruplicaram as participações nas capacitações deste tema.

Nos meses onde o indicador de infecção começa a diminuir, em 2017, paralelamente, as participações aumentaram, mostrando esta possível interferência, evidenciada pela instituição, posteriormente, no relatório de gestão.

Esses resultados podem não comprovar a interferência de forma conclusiva, mas podem indicar que a educação em saúde ajudou na redução do indicador.

Porém, é claro que as ações educativas por si só, realizadas de forma isolada, não são as únicas responsáveis pela mudança no processo de trabalho, onde uns conjuntos de outras ações também foram importantes na construção dos resultados do indicador.

Por fim, considera-se que esta pesquisa foi importante para a gestão em saúde, pois a infecção hospitalar é um fator que causa altos índices de mortalidade, aumenta a permanência na internação hospitalar e, consequentemente, eleva os custos com os pacientes. Precisamos saber quais metodologias são capazes de auxiliar os gestores na busca por melhores práticas e melhores resultados na assistência e, a partir deste trabalho de pesquisa, podemos dizer que a educação é uma ferramenta importante na transformação do trabalho dos profissionais de saúde. 


\section{Referências}

1. Santos, Adélia Aparecida Marçal Dos; Et Al. Fortalecendo O Desempenho Hospitalar Em Brasil. In: Programa De Pesquisas Hospitalares Em Busca De Excelência: Diagnóstico Do Controle Da Infecção Hospitalar No Brasil. [Publicação Online]; 2005.[ Acesso Em 15 Maio 2018]. Disponível Em: Http://Www.Anvisa.Gov.Br/Servicosaude/Co ntrole/Infectes\%20hospitalares_Diagnostico.P $\underline{\mathrm{df}}$

2. Fernandes, V.F.; Et Al. Análise Da Taxa De Infecção Hospitalar Em Um Hospital Universitário Do Rio Grande Do Sul. Congresso Brasileiro De Medicina Hospitalar; Congresso Gaúcho De Clínica Médica. Ii E Xii. 2014. Porto Alegre. São Paulo: Editora Blucher, V.1, N.5, 2014. P.15. [Acesso Em 16 Maio 2018]. Disponível Em Http://Pdf.Blucher.Com.Br.S3-Sa-East1.Amazonaws.Com/Medicalproceedings $/ 2 \mathrm{cb}$ $\underline{\mathrm{mh} / 006 . \mathrm{Pdf}}$

3. Brasil. Empresa Brasileira De Serviços Hospitalares (Ebserh). Protocolo De Prevenção De Infecção De Corrente Sanguínea; 2016. [Acesso Em 15 Maio 2018]. Disponível Em: Http://Www.Ebserh.Gov.Br/Documents/2202 50/1649711/Protocolo+Preven\%C3\%A7\%C3 $\%$ A3o+Ics.Pdf/B5d860cb-E3a9-4585-B039$\underline{86 \mathrm{bf} 1202 \mathrm{dcc} 7}$

4. Gigante, Renata L.; Campos, Gastão W. De S.; Política De Formação E Educação Permanente Em Saúde No Brasil: Bases Legais E Referências Teóricas. Trabalho, Educação \& Saúde, Rio De Janeiro, V. 14 N.3. 2016. P.747-763. Disponível Em: Http://Www.Scielo.Br/Pdf/Tes/V14n3/16781007-Tes-14-03-0747.Pdf Acesso Em: 8 Ago 2018.

5. Brasil. Sistema Único De Assistência Social Suas. Norma Operacional Básica De Recursos Humanos Do Suas. [Publicação Online]; Brasília, Dezembro De 2006. [Acesso Em 4 Set 2018]. Disponível Em: Http://Www.Assistenciasocial.Al.Gov.Br/Sala -De-Imprensa/Arquivos/Nob-Rh.Pdf

6. Ceccim, Ricardo B.; Educação Permanente Em Saúde: Descentralização E Disseminação De Capacidade Pedagógica Na Saúde. Ciência \& Saúde Coletiva [Revista Em Internet]. Rio De Janeiro, Rj, V. 10 N.4. 2005. [Acesso Em 4 Ago 2018]. Disponível Em: Http://Www.Scielo.Br/Pdf/Csc/V10n4/A20v1 $\underline{\text { On4.Pdf }}$
7. Brasil. Anvisa. Agência Nacional De Vigilância Sanitária. Boletim Segurança Do Paciente E Qualidade Em Serviços De Saúde $N^{\circ}$ 16: Avaliação Dos Indicadores Nacionais Das Infecções Relacionadas À Assistência À Saúde (Iras) E Resistência Microbiana Do Ano De 2016. Gvims/Ggtes/Anvisa. [Publicação Online]; Dezembro De 2017. [Acesso Em 05 Nov 2018]. Disponível Em: Https://Www20.Anvisa.Gov.Br/Segurancadop aciente/Index.Php/Publicacoes/Item/BoletimSeguranca-Do-Paciente-E-Qualidade-EmServicos-De-Saude-N-16-Avaliacao-DosIndicadores-Nacionais-Das-InfeccoesRelacionadas-A-Assistencia-A-Saude-Iras-E$\underline{\text { Resistencia-Microbiana-Do-Ano-De-2016 }}$

8. Mendonça, Katiane M.; Neves, Heliny C. C.; Barbosa, Divina F. S.; Et Al. Atuação Da Enfermagem Na Prevenção De Controle De Infecção De Corrente Sanguínea Relacionada A Cateter. Revista Enfermagem [Revista Em Internet]. Uerj. Rio De Janeiro. V. 19. No 2. 2011. [Acesso Em 07 Nov 2018]. Disponível Em:

Http://Www.Facenf.Uerj.Br/V19n2/V19n2a26 $\underline{\text { Pdf }}$

9. Santos, Saymom Fernando. Et Al. Ações De Enfermagem $\mathrm{Na}$ Prevenção De Infecções Relacionadas Ao Cateter Venoso Central: Uma Revisão Integrativa. Revista Da Associação Brasileira De Enfermeiros De Centro Cirúrgico - Sobecc [Revista Em Internet]. São Paulo. V.19. N. 4. 2014. P. 219225. [Acesso Em 07 Nov 2018]. Disponível Em:

Http://Sobecc.Org.Br/Arquivos/Artigos/2015/ Pdfs/V19n4/Sobecc_V19n4_219-225.Pdf

10. Brasil. Anvisa. Agência Nacional De Vigilância Sanitária. Medidas De Prevenção De Infecção Relacionada À Assistência À Saúde. [Publicação Online]; Brasília: Anvisa, 2017. [Acesso Em 05 Nov 2018]. Disponível Em:

Http://Portal.Anvisa.Gov.Br/Documents/3385 2/3507912/Caderno+4+-

+ Medidas+De+Preven $\%$ C3\% A7\%C3\%A3o+ De+Infec\%C3\%A7\%C3\%A3o+Relacionada+ \%C3\%A0+Assist\%C3\%Aancia+\%C3\%A0+S a\%C3\%Bade/A3f23dfb-2c54-4e64-881cFccf9220c373

11. Gil, Antônio Carlos. Como Elaborar Projetos De Pesquisa. São Paulo: Atlas, 2002. 4. Ed.

12. Brasil. Ministério Da Educação - Mec. Secretaria De Educação Superior - Sesu/Mec. Prestação De Contas Ordinárias Anual. Relatório De Gestão Do Exercício De 2016. Porto Alegre - Maio 2017. 
13. Brasil. Ministério Da Educação - Mec. Secretaria De Educação Superior - Sesu/Mec. Prestação De Contas Ordinárias Anual. Relatório De Gestão Do Exercício De 2014. Porto Alegre - Maio 2015.

14. Brasil. Ministério Da Educação - Mec. Secretaria De Educação Superior - Sesu/Mec. Prestação De Contas Ordinárias Anual. Relatório De Gestão Do Exercício De 2015. Porto Alegre - Março De 2016.

15. 15. Brasil. Ministério Da Educação - Mec. Secretaria De Educação Superior - Sesu/Mec. Prestação De Contas Ordinárias Anual. Relatório De Gestão E Administração Do Exercício De 2017. Porto Alegre, Fevereiro De 2018.

16. Oliveira, Francimar Tinoco De; Et Al. Comportamento Da Equipe Multiprofissional Frente Ao Bundle Do Cateter Venoso Central $\mathrm{Na}$ Terapia Intensiva. Escola Anna Nery
Revista De Enfermagem [Revista Em Internet]. V.20. N.1. 2016. P.55-62. [Acesso Em 15 Maio 2018]. Disponível Em: Http://Www.Scielo.Br/Pdf/Ean/V20n1/14148145-Ean-20-01-0055.Pdf

17. Brasil. Ministério Da Educação - Mec. Secretaria De Educação Superior - Sesu/Mec. Comissão De Controle De Infecção Hospitalar. Relatório Dos Indicadores De Infecção Hospitalar. Dezembro 2017.

18. Brasil. Ministério Da Educação - Mec. Secretaria De Educação Superior - Sesu/Mec. Comissão De Controle De Infecção Hospitalar. Relatório Dos Indicadores De Infecção Hospitalar. Dezembro 2016.

\section{Participação dos autores:}

Silveira JS trabalhou na concepção teórica, coleta de dados, análise estatística e elaboração e redação final do texto.

Nugem RC trabalhou na orientação, desenvolvimento dos objetivos e da metodologia, na redação e na revisão final do texto.

Recebido: 30.07.2019

Revisado: 09.08.2019

Aprovado: 13.09.2019 